\title{
Interfacial Characteristics of Space Charge in Multi-layer LDPE
}

\author{
Z. $\mathrm{Xu}^{*}$ and G. Chen \\ School of Electronics and Computer Science, University of Southampton, SO17 1BJ UK \\ *E-mail : zx04r@ecs.soton.ac.uk
}

\begin{abstract}
This paper reports on the investigation into space charge characteristics at interfaces of multi-layer polymeric materials. Planar low density polyethylene (LDPE) samples were used in this study. DC voltage up-to $10 \mathrm{kV}$ was applied to the sample and space charge measurements were taken using the pulsed electro-acoustic (PEA) technique. Experimental results indicated that the electrode interface plays an important role in determining the bulk charge formation and polymer layer interface has different effects on electrons and holes trapping.
\end{abstract}

Index Terms--space charge, interface, LDPE, PEA

\section{INTRODUCTION}

$\mathrm{I}_{\mathrm{s}}^{\mathrm{n}}$ nterfaces are often encountered in practical insulation systems and have become a growing area of interest due to their influence on the electrical performance of whole system. A dielectric material usually can store charge on its surface after the charge exchange occurring at the interface contact with other dielectrics. In electrostatics, the stored charge may be the source of electrostatic discharge and electromagnetic damage for equipments. Electrical effects at interfaces and surfaces in polymeric dielectrics have received a lot of attentions because most of breakdowns happen at the interfaces [1]. The interfaces in high electrically stressed materials, whether polymer/metal or polymer/polymer, can cause accumulation of space charge which can lead to unwanted electric field modification. In high voltage transmission power cables, interfaces between the dielectric and the electrode are weak points for electrical insulating [2]. For example, the interfaces in cable joints and terminations of extruded high voltage cables have been identified as crucial parts as their failure probability is much higher than cable itself.

It has been proposed that the accumulated space charge at the interface is a more significant factor with regards to electric field enhancement than in the bulk [3]. Despite of increasing interests in the interfaces, the underlying mechanisms related to charge formation and electrical ageing are not well understood and research into the best interfacial materials and its binding structure is becoming increasingly important.

Space charge behaviours are complicated and sensitive to many factors, such as dielectric structure, applied field and temperature. More work is required to be done on interfacial charge behaviour, including interface conditions and different applied electric fields. In our previous paper [4] where space charge behaviour in two layers of polyethylene films has been studied, in the present research we have extended our research to three layers of LDPE films. Three different thickness
$(50 \mu \mathrm{m}, 100 \mu \mathrm{m}$, and $180 \mu \mathrm{m})$ planar additive-free LDPE films were measured under dc electric stress for an extended period of time. Space charge was measured using the pulsed electro-acoustic (PEA) technique. Particular attention was paid to the interfaces.

\section{EXPERIMENTAL DETAILS}

\section{A. Sample information}

The formation of space charge in the dielectric material is greatly affected by the presence of impurities and additives, which can act as ionisable centres or trap sites under high electric field. In order to reduce the influence of impurities, additive-free low-density polyethylene film was chosen in this study. Three kinds of thickness film $(50 \mu \mathrm{m}, 100 \mu \mathrm{m}$, and $180 \mu \mathrm{m})$ were purchased from the Goodfellow. The thickness of samples in this study is $\sim 200 \mu \mathrm{m}$. Because the dispersion influence of material is not significant in thin films, it is not considered in this study. The electrodes used in this study were aluminium (Al) and semi-conducting polymer (Sc). In the case of semicon electrode material, thin film $(<100 \mu \mathrm{m})$ was made from semicon pellet by a hot pressing process. The semicon pellet was made of cable grade polyethylene loaded with carbon black to increase conductivity (Borealis LOE592)

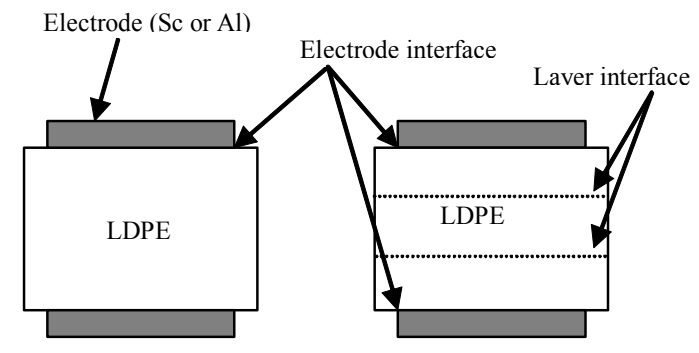

Fig.1: Schematic diagram of sample arrangement.

A sample structure of interface is shown in Fig.1. For easy description in later sections, here the two types of interface are named as layer interface (polymer/polymer interface) and electrode interface (electrode/polymer interface).

\section{B. Measurement system}

In this study, space charge distribution characteristic was measured by the PEA method, which is by far the most widely used technique in the field of space charge measurements. 
This technique utilizes the interaction between high voltage pulses and charge layers accumulated in the insulating material to produce acoustic pressure waves, which traverse across the material. Over the main points, acoustic pressure waves are produced when an electrical pulse, applied externally, interacts with charge layers at the electrodes and/or in the material. The acoustic waves, which are proportional to the charge at the layers, are converted into an electrical signal by a piezo-electric transducer, amplified and captured with a digital oscilloscope. Details on the principle of PEA can be found in [5].

For a PEA system, the spatial resolution is determined by several factors such as pulse width, acoustic speed in the sample, and the sensor thickness. In our measurement system, an electric pulse of $2 \mathrm{~ns}$ duration and $0.6 \sim 1 \mathrm{kV}$ amplitude is applied to the sample to generate the acoustic signal. The sensor used was a $4 \mu \mathrm{m}$ thick PVDF material. This gives a spatial resolution of about $\sim 4 \mu \mathrm{m}$.

\section{RESULTS AND DISCUSSION}

\section{A. One layer sample}

In order to understand the space charge characteristic at the interface of dielectrics, investigation on bulk charge distribution was done first. Fig.2 shows the space charge distribution obtained from $180 \mu \mathrm{m}$ LDPE film with semicon film as the anode and $\mathrm{Al}$ as the cathode during 2hours stressing period.

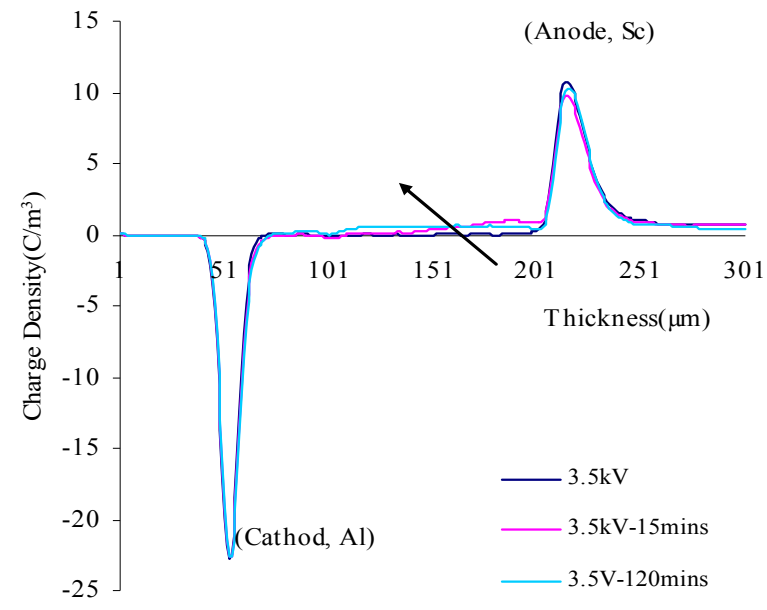

Fig.2: Space charge profiles of single layer sample $(180 \mu \mathrm{m})$.

Any charge measured in the bulk should not be considered as ionization because the additive-free low density polyethylene sample is used here. The most possible reason for charge formation is injected charge from the electrodes. From the early work we know that the charge distribution in the bulk strongly depends on the electrode materials $[6,7]$. Generally, Sc injects more charge than $\mathrm{Al}$ electrode. The results shown in Fig.2 follow the same pattern. During the first part of stressing (15mins), positive charge injected from the anode can be seen in the region close to the anode. As the stressing time becomes longer, the positive charge starts to move towards the cathode under the influence of the applied electric field and dominates majority of the bulk region. On the other hand, there is less negative charge observed in the region adjacent to the cathode. There may be two reasons for this phenomenon. Firstly, Al electrode may inject less charge compared with Sc. Secondly, the injected charge may be present together with the positive charge, but the PEA technique only shows the net charge.

\section{B. Two layer sample}

Fig.3 illustrates the space charge formation for the two-layer LDPE sample $(100 \mu \mathrm{m}$ and $100 \mu \mathrm{m})$. Similar to the one layer $3.5 \mathrm{kV}$ was applied to the two layer sample. Observing the layer near to the anode, we can see the progress of positive charge injection into the bulk of sample and moves towards the cathode. The charge dynamics in the region close to the cathode is slightly different. Initially, the negative charge starts to appear followed by a gradual reduction. After $15 \mathrm{mins}$ stressing, more and more positive charge moves into this region and finally overtakes the negative charge. At the polymer interface, the most attractive area for this research, charge accumulation pattern changes with stressing time. In the first hour, negative charge build-up increases with time. From the results it can be seen that a significant amount of negative charge has been accumulated at the interface. In the second hour of stressing, however, the negative charge starts to decreases gradually. At the end of the stressing period, there is only a small amount of negative charge present at the interface.

The above observed phenomena may be attributed to the fact that the semicon film chosen as the anode helped the charge injection into the LDPE sample.

According to the proposed explanation in [7], once the external voltage is applied across the sample, charge injection takes place. Charge injection from the anode may dominate due to Sc electrode, forming positive charge in the region adjacent to the anode. The electric field is modified by the presence of the positive charge and the electric field in the region close to the cathode will be enhanced represented by

$$
\mathrm{E}_{\text {total }}=\mathrm{E}_{\text {applied }}+\mathrm{E}_{\text {space charge }}
$$

The resultant high electric field at the cathode will enhance charge injection from the cathode and the injected electrons from cathode will move towards to the anode under the influence of the electric field. The electrons encounter the layer interface and are trapped at the interface. From the charge distribution shown in Fig. 3, it seems that it is easier for electrons to be trapped at the interface than positive charge carriers. That is to say the positive charge can pass through the interface and move to next layer.

The reduction of negative charge at the layer interface over a long period of time deserves an explanation. It could be one of the following two mechanisms. First, the electrons trapped at the interface may be not deeply trapped, and over a period 
of time they may release themselves from the traps. Alternatively, the electrons are deeply trapped at the interface, however, the electric field due to the presence of negative charge at the interface may cause electric field enhancement in the region close to the anode. This will certainly lead to more positive charge injection. The movement of positive charge towards the cathode will meet with the interface. Consequently, the resultant charge at the interface, which is measured by the PEA, shows a reduction, either via simple sum or recombination between positive and negative charges.

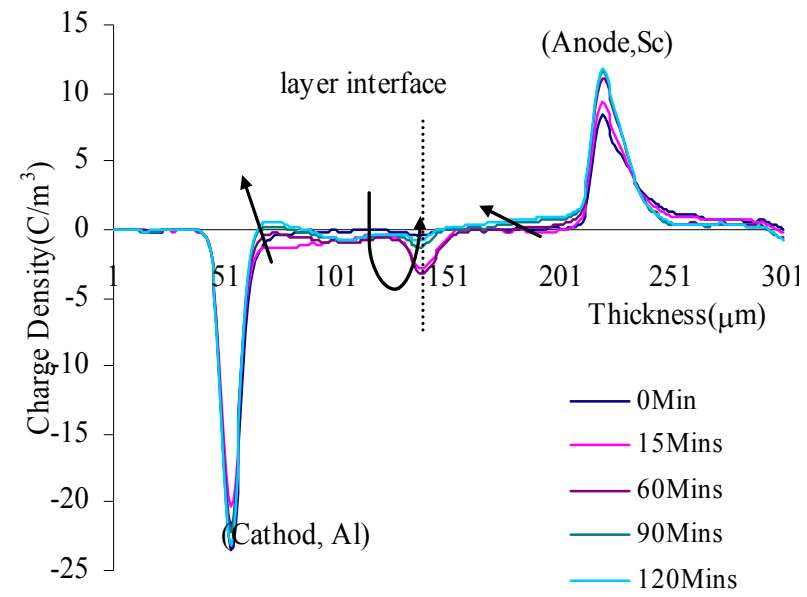

Fig.3: Space charge profiles of two-layer sample $(100 \mu \mathrm{m}+100 \mu \mathrm{m})$.

\section{Three layer sample}

Fig.4 shows space charge distribution in the three-layer sample. To maintain a similar thickness, we use a balance combination multi-layer sample, same thickness film $(50 \mu \mathrm{m})$ attached to the middle layer $(100 \mu \mathrm{m})$. Fig. 4 shows clearly that positive charge appeared in the bulk of every layer and negative charge happened at the interface close to the cathode even under low electric field $(10 \mathrm{kV} / \mathrm{mm})$.

When the applied voltage is increased to $5 \mathrm{kV}$, the charge distribution in the multi-layer is illustrated in Fig. 5. The charge at the layer interface next to the anode is positive charge in this case, a peak is observed there. Charge in the bulk and charge at the interface next to the cathode show the same distributions as Fig. 4.

This three layer LDPE sample was tested under $10 \mathrm{kV}$ electric stress and over a long period of stressing. The results of space charge distribution are shown in Fig.6. The bulk charge polarity in every layer is positive and the negative peak can be found only at the interface close to the cathode. A very interesting observation is that the negative charges accumulated at the layer interface close to the cathode increase to the maximum value in $10 \mathrm{mins}$ and then decrease. This is similar to the interface results in two layer sample (Fig.3), which need 60mins stressing to reach the maximum value under lower electric field. It seems to suggest that the higher electric field can accelerate charge movement. At the same time, the positive charge in the sample bulk decreases. After 10mins stressing under the high electric field $(50 \mathrm{kV} / \mathrm{mm})$, the net charge accumulate at the layer interface close to the anode was negative. After 1hour stressing the charge distribution reaches to a relative stable situation, the results of $60 \mathrm{mins}$ and results of $90 \mathrm{mins}$ nearly overlapped.

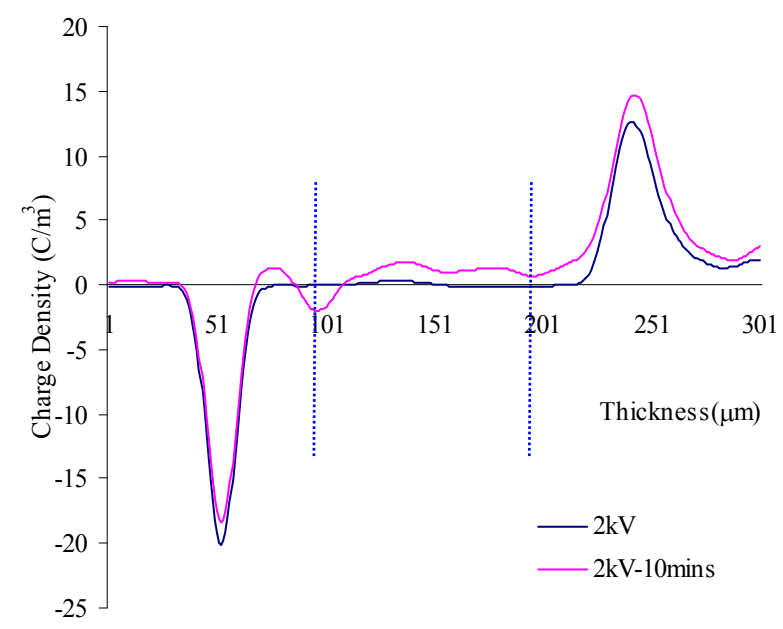

Fig. 4: Space charge profiles of three-layer LDPE sample $0 \mu \mathrm{m}+100 \mu \mathrm{m}+50 \mu \mathrm{m})$ under $2 \mathrm{kV}$.

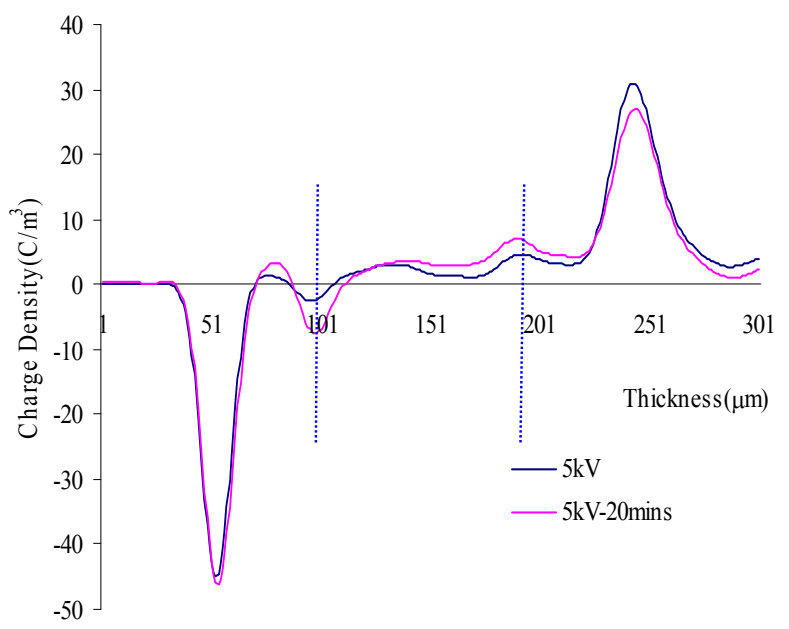

Fig. 5: Space charge profiles of three-layer LDPE sample $0 \mu \mathrm{m}+100 \mu \mathrm{m}+50 \mu \mathrm{m}$ ) under $5 \mathrm{kV}$.

By testing the space charge distribution in the multilayer LDPE sample with different electric field and different period, it is evident that injection takes place at both electrodes. Positive charge from semicon electrode can inject into the bulk more easily than the electrons from Al electrode. These positive carriers tend to move towards the cathode under the influence of electric field and at the same time the injected electrons have a propensity to move to the anode. When these two kinds of charge meet at the polymer interface under the low electric field $(10 \mathrm{kV} / \mathrm{mm})$, it seems that electrons can be trapped while positive charges travel through (see Fig.4). But the positive charge can be blocked by the polymer interface when an appropriate electric field $(25 \mathrm{kV} / \mathrm{mm}$, see Fig.5) is applied. The polymeric interface acts as a barrier for electrons 
because the negative peak at the interface was found unproblematic for multilayer examination. On the other hand, the layer interface has little effect on positive charge because they can reach the layer next to the cathode.

It is believed that recombination between electrons and positive charge takes place throughout the entire sample. The PEA technique only gives us the resultant charge or net charge. Further investigation should be done to understand the exact mechanism and situation at the interface of polymeric materials.

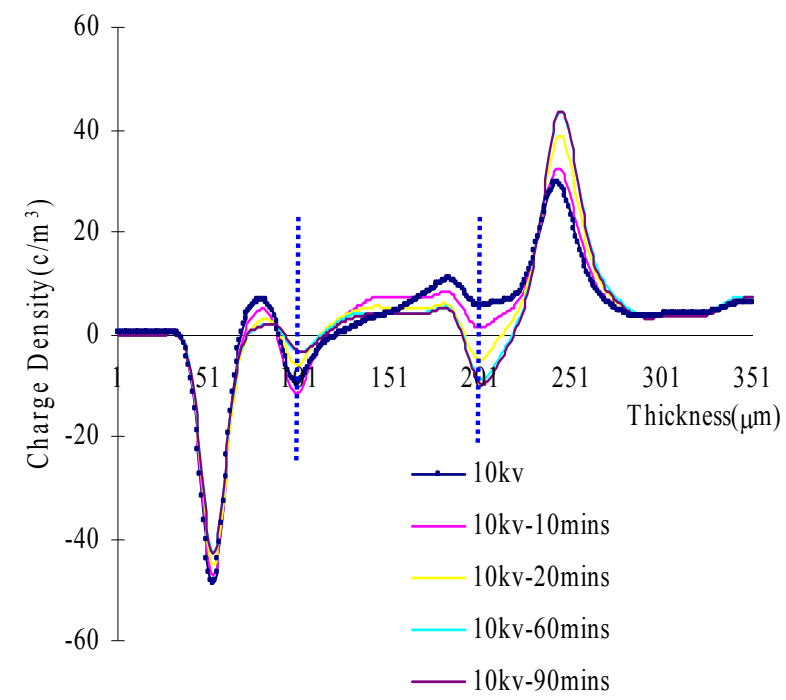

Fig. 6: Space charge profiles of three-layer LDPE sample $0 \mu \mathrm{m}+100 \mu \mathrm{m}+50 \mu \mathrm{m}$ ) under $10 \mathrm{kV}$.

\section{CONCLUSION}

Space charge distribution in multilayer LDPE samples with different electrode materials using the PEA technique has been reported. From the results and discussion, the following conclusions can be drawn.

The electrode material has an effect on space charge injection and trapping characteristics. Positive charges from semicon anode inject into the bulk easily and travel to the opposite electrode. The interface between LDPE films acts as traps both for electrons and positive charge carriers, especially for the electrons. After longer ageing period or using higher electric field, electrons can escape from barrier and keep move into the bulk. They will trap at the next interface and reach to another relative stable situation.

\section{REFERENCES}

[1] T. J. Lewis, "Electrical Effect at Interfaces and Surfaces", IEEE Trans. Elcctr. Insul, Vol. 21, pp. 289-295, 1986.

[2] Y. Li and T. Takada, "Space Charge Distribution in Multi-Ply LDPE", Electrical Insulation and Dielectric Phenomena. Annual Report, Victoria, BC, Canada, pp. 397-402, 1992.

[3] Y. Li, T. Takada, H. Miyata and T. Niwa, "Observation of Charge Behavior in Multiply Low-Density Polyethylene", J.Appl.Phys., vol. 74, pp. 2725-2730, 1993.

[4] Z. Xu and George Chen, "Space Charge Behaviour at Ldpe Interface under Ac Electric Stress", The 6th international space charge conference, 2006.
[5] T. Maeno, T. Futami, H. Kushibe, T. Takada and C. M. Cooke, "Measurement of Spatial Charge Distribution in Thick Dielectrics Using the Pulsed Electroacoustic Method", IEEE Transactions on Electrical Insulation, Vol. 23, pp. 433-439, 1988.

[6] George Chen, "Interfaces and Space Charge in Polymeric Insulating Materials", Materials Research Society, vol. 889, pp. w08-03.01-w08-03.12, 2006.

[7] George Chen, Y.Tanaka, T. Takada and L. Zhong, "Effect of Polyethylene Interface on Space Charge Formation", IEEE Trans. DEI, Vol. 11,pp. 113-121, 2004. 